\title{
Zur Geschichte der Geowissenschaften im Museum für Naturkunde zu Berlin Teil 1: Aus der Vorgeschichte bis zur Gründung der Berliner Bergakademie im Jahre 1770
}

\author{
Günter Hoppe $^{1}$
}

Mit 3 Textabbildungen

\section{Zusammenfassung}

Die Geschichte der beiden Institute für Mineralogie und für Paläontologie des Berliner Museums für Naturkunde mit ihren sehr großen Sammlungen beginnt mit ihrer direkten Vorgängerin, der Berliner Bergakademie, die 1770 gegründet worden ist. Aber bereits vor dieser Zeit hat es in Berlin geowissenschaftliche Interessen und Betätigungen gegeben.

Diese Vorgeschichte wird mit einer Zeit begonnen, in der es den Ort Berlin noch längst nicht gab. Aus der La-Têne-Zeit, die der Zeit der griechischen Antike entspricht, stammt eine Aschenurne mit einer Sammlung fossiler Mollusken, die im norddeutschen Flachland bei Bernburg gefunden wurde. Die Zusammensetzung dieser Sammlung läßt bereits ein wissenschaftliches Herangehen erkennen.

Für Berlin selbst ist kurz nach Georg Agricola eine Persönlichkeit der Renaissance zu verzeichnen, Leonhard Thurneysser zum Thurn, in dessen vielfältigen Aktivitäten auch Mineralien einen Platz hatten. In gleicher Zeit war in Berlin am brandenburgischen Hofe eine Raritätenkammer vorhanden, die spätere Kunst- und Naturalienkammer. Sie existierte bis über das Jahr 1770 hinaus und enthielt auch Mineralien und Versteinerungen. Das sich hierdurch zeigende Interesse an solchen Objekten war noch recht oberflächlich.

Erst die Sammlungen privater Personen, die in Berlin seit Ende des 17. Jahrhunderts entstanden sind, zeigen ein tieferes und wissenschaftliches Interesse, wenn auch in verschiedenem Maße und in unterschiedlicher Spezialisierung. Unter ihnen ragt besonders Johann Gottlob Lehmann heraus. Als vielseitiger Naturwissenschaftler und Bergrat hielt er privat Vorlesungen in Mineralogie und Bergbaukunde. Der Siebenjährige Krieg verhinderte den Ausbau und die Fortsetzung.

Erst Jahre danach, 1768, reorganisierte König Friedrich II. das preußische Bergwesen und richtete 1770 die Berliner Bergakademie ein. Hierbei kam dem Arzt und Bergrat Carl Abraham Gerhard bei der Einrichtung und als Lehrkraft eine wesentliche Rolle zu.

\begin{abstract}
History of the Geoscience Institutes of the Natural History Museum in Berlin. Part 1.

The Geoscience Institutes of the Natural History Museum in Berlin have their roots in the Mining Academy which was founded in 1770. Geoscientific interest, however, goes back as far as to prehistoric times which is, e.g., evidenced by a collection of mollusks from the Iron Age. From the Renaissance, similar interests were developed by Leonhard Thurneysser zum Thurn. The contemporaneous cabinet of arts and curiosities of the Prussian Dynasty is also known to have housed geoscientific pieces which, however, turned out to be of only subordinate significance later. Much more important were the efforts of Berlin citizens in the $17^{\text {th }}$ and $18^{\text {th }}$ century who established remarkable collections of geoscientific objects. Among these collectors, Johann Gottlob Lehmann was the most outstanding personality. He gave not only lectures but also wrote textbooks on geoscientific topics. However, not before the end of the Seven Years-War Carl Abraham Gerhard was authorized to found the Mining Academy.
\end{abstract}

\section{Einleitung}

Das Berliner Museum für Naturkunde ist als naturhistorisches Forschungsinstitut mit seinen Sammlungen geowissenschaftlicher und zoologischer Objekte ein Zentralinstitut der HumboldtUniversität, das die drei Institute für Mineralogie, für Paläontologie und für Systematische Zoologie umfaßt. Es ist untergebracht in dem
1883 bis 1888 erbauten Museumsgebäude in Berlin-Mitte, Invalidenstraße 43.

Die beiden geowissenschaftlichen Institute des Museums für Naturkunde gehen gemeinsam mit ihren Sammlungen direkt und in lückenloser Folge auf die Lehranstalt des preußischen Bergund Hüttendepartements, die Berliner Bergakademie, zurück, die 40 Jahre früher als die Berliner Universität im Jahre 1770 gegründet wurde.

\footnotetext{
1. Museum für Naturkunde, Institut für Mineralogie, Invalidenstr. 43, D-10115 Berlin, Germany. Erhalten Januar 1998, angenommen Juli 1998
} 
Ihre Lehr- und Forschungssammlung entwickelte sich als Königliches Mineralienkabinett zu einer bedeutenden Sammlung, die 1801 zusammen mit anderen Institutionen ein eigens erbautes Haus, die sogenannte Neue Münze, erhielt, wo sie auch in musealer Weise genutzt wurde. Im Jahre 1810 gelangte das Königliche Mineralienkabinett entsprechend der Konzeption Wilhelm von Humboldts einschließlich der Lehrfunktion der Bergakademie als Mineralogisches Museum in die neugegründete Berliner Universität und wurde 1813 in das Gebäude der Universität, Unter den Linden, überführt.

Bereits im Königlichen Mineralienkabinett enthielten die Sammlungen nicht allein Minerale im heutigen Sinn, sondern, dem damaligen weiten Umfang des Mineralogiebegriffes entsprechend, geowissenschaftliche Sammlungsobjekte aller Art, wobei allerdings zunächst das Hauptaugenmerk auf die Minerale im engeren Sinn gerichtet war. Die Entwicklung anderer Zweige der Geowissenschaften und damit auch die darauf ausgerichtete Spezialisierung der Sammlungen begann erst allmählich, machte aber im Laufe des 19. Jahrhunderts, vor allem mit der Herausbildung der Paläontologie, große Fortschritte. Dies hatte die Konsequenz des verstärkten Ausbaues der Sammlungen und des erhöhten Raumbedarfs, was sich zu einem dringenden Problem im Universitätsgebäude entwickelte und schließlich durch den Bau des Gebäudes für das Museum für Naturkunde gelöst wurde. Der entstandenen Spezialisierung wurde dadurch Rechnung getragen, daß das Mineralogische Museum beim Bezug des neuen Gebäudes in zwei Teile getrennt wurde, in das Institut für Mineralogie und Petrographie sowie in das Institut für Geologie und Paläontologie mit den jeweiligen Sammlungen. Sie sind die Vorstufen der heutigen zwei geowissenschaftlichen Institute des Museums für Naturkunde.

Im folgenden soll zunächst die vor der Gründung der ersten geowissenschaftlichen Vorläuferinstitution liegende Vorgeschichte von den frühesten Wurzeln an behandelt werden.

\section{Prähistorische Wurzeln}

Das Interesse an Naturobjekten, die wir heute zu dem Bereich der Geowissenschaften zählen (im folgenden: geowissenschaftliche Objekte), hat sich bereits in sehr frühen Zeiten der Menschheitsgeschichte entwickelt. Es war an Eigenschaften dieser Objekte geknüpft, die die Verwendung bzw. Herrichtung als Waffen und Werkzeuge so- wie als Schmuck oder als Grundstoffe für Keramik und Metallgewinnung ermöglichten. Zeugnisse für ein darüber hinausgehendes Interesse, das nicht direkt mit der Nützlichkeit bzw. unmittelbaren Verwendung der Objekte zusammenhängt und gegebenenfalls geradezu wissenschaftlich genannt werden könnte, gibt es nur selten.

Ein besonders überzeugendes Beispiel für letzteres aus dem norddeutschen Flachland, in dem auch Berlin liegt, bietet ein Fund von Groß-Wirschleben bei Bernburg an der Saale (Merkel 1904), der aus der La-Tène-Zeit stammt (frühe Eisenzeit, vor etwa 2500 bis 2000 Jahren). Dieser Fund besteht aus einer Aschenurne mit der Beigabe einer Sammlung von über 50 Molluskenschalen, vorwiegend Gehäusen von (Turm-) Schnecken und einigen Muschel- und Grabfüßlerschalen. Die Schneckengehäuse ähneln sich untereinander, gehören aber mehreren Arten an. Eine moderne Untersuchung (Tembrock 1982) bestätigte und präzisierte, daß die Sammlung, von zwei rezenten Exemplaren abgesehen, mit zahlreichen fossilen Arten aus verschiedenen Stufen des Tertiärs bestückt ist, die größtenteils aus der Umgebung des Fundortes stammen können. Es sind aber immer nur ein oder zwei Exemplare jeder Art in der Sammlung vertreten, gleichgültig wie häufig die betreffenden Arten in den Tertiärsedimenten vorkommen. Einige Arten sind höchst selten zu finden und einige wenige kommen sogar in der Umgebung überhaupt nicht vor, dafür aber in Südfrankreich bzw. am Niederrhein. Man muß deshalb annehmen, daß die Sammlung von einem genauen, hoch interessierten Beobachter mit groBem Bedacht zusammengetragen worden ist, zum Teil wohl auch auf Reisen oder auf dem Tausch- oder Kaufwege.

Die La-Tène-Zeit, in der die Sammlung entstanden ist, fällt etwa mit der Entwicklungszeit der hochstehenden Kultur des griechischen Altertums am Mittelmeer zusammen. Von den griechischen Philosophen wissen wir aus ihren überlieferten Schriften, daß sie die Natur aufmerksam beobachtet haben. Am umfangreichsten sind Nachrichten hierüber in den Schriften des Atheners Theophrastos, der von 372 bis 286 v. u. Z. gelebt hat, zu finden, eines Schülers und Freundes von Aristoteles. In seiner Schrift über die Steine (peri lithon) stellte Theophrastos Kenntnisse über die damals bekannten und verwendeten Gesteine und Minerale, wie Bau- und Bildhauergesteine, Erze, Schmucksteine und andere, zusammen und machte Angaben über ihre Herkunft, Verwendung und Eigen- 
schaften (Schmieder 1807; Lenz 1861). Derart detailliertes, wie wir es aus dem Bernburger Fund kennen und herauslesen, ist aus der Antike jedoch nicht überliefert. Theophrastos nennt auch nur sehr wenige Versteinerungen organischer Entstehung (Korallen, Luchsstein, Bernstein), auch in seiner Schrift über die Tiere (Lenz 1856) findet man an fossilen Tierresten nur Elephantenstoßzähne. Gleiches trifft im wesentlichen auch auf die 31느 Jahrhunderte späteren Darstellungen des Römers Plinius d. Ä. (23-78 u. Z.) zu. Andererseits sind im griechischen und römischen Altertum durchaus Sammlungen und Schatzkammern erwähnt worden, die unter anderem auch Minerale enthielten (Niedermayr 1988).

Von den frühen Zeugnissen des Interesses an steinernen Naturobjekten muß man sehr weit in der Geschichte der Menschheit voranschreiten, bis sich nach den Stürmen der Völkerwanderung und der folgenden Stagnation etwas zeigt, das einen neuen Anfang darstellt. Der Neubeginn und das Anknüpfen an die Kenntnisse der Antike wurden gestört durch das Aufkommen mystischer Vorstellungen und auch durch die Annahme magischer Kräfte, die u. a. Edelsteinen zugeschrieben wurden, sowohl in der Heilkunde wie in der entstehenden Alchemie. Die ersten wahren Fortschritte stellten sich ein, als der Bergbau, besonders im Erzgebirge und im Harz, in voller Blüte stand und sich im Zeitalter der Renaissance und des Humanismus eine freiere Geisteshaltung durchsetzte. Es war die Wirkungszeit des sächsischen Arztes und mehrmaligen Chemnitzer Bürgermeisters Georgius Agricola (1484-1555), der zugleich und vor allem ein berühmter Bergbaukundiger und Mineraloge war. Er fußte auf den antiken Kenntnissen über die Steine und verband dies mit seinen eigenen Erfahrungen, die er im erzgebirgischen Bergbau als Arzt und kritischer Beobachter der Natur und der Technik gewonnen hatte. In einigen seiner Schriften legte er dies nieder (Agricola 1530, $1546,1556)$. Sie sind so bedeutend, daß sie in heutiger Zeit neu herausgegeben worden sind (u. a. Prescher 1955/92). Daneben betätigte sich Agricola auch als Sammler von Mineralen. Andere folgten ihm darin nach, wie zum Beispiel der Pfarrer Johannes Mathesius (1504-1565) in St. Joachimsthal (Prescher 1955; Bartnick 1992).

\section{Leonhard Thurneysser zum Thurn in Berlin}

Für Berlin sind die ersten, durch Überlieferungen nachgewiesenen Anzeichen des Interesses

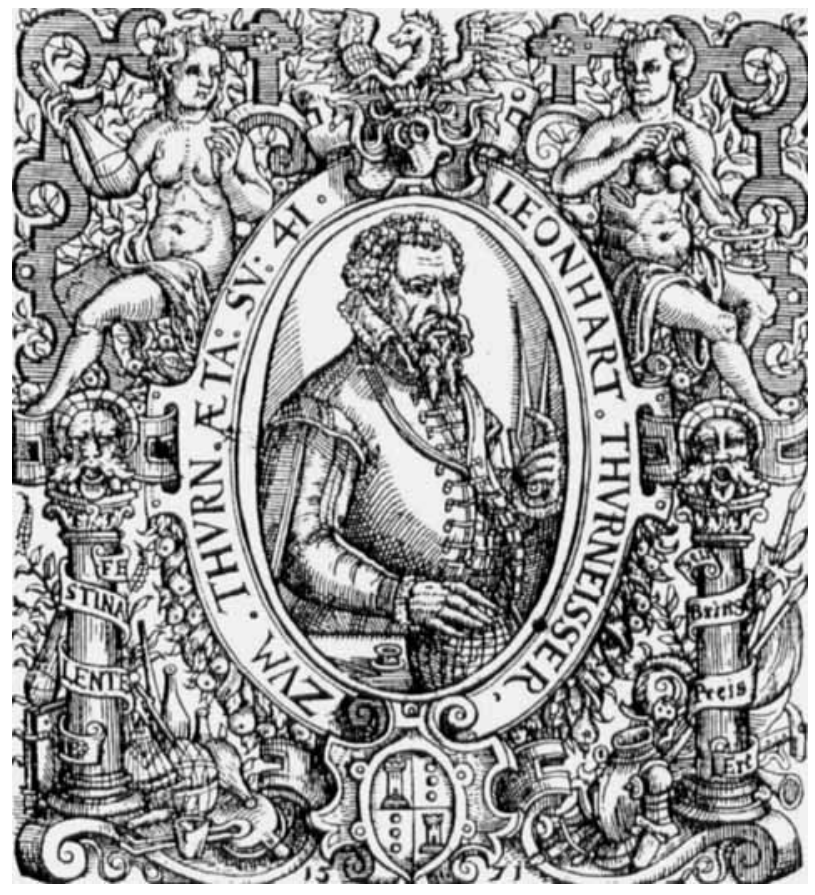

Abb. 1. Leonhard Thurneysser zum Thurn (1531-1596). Porträt im Alter von 41 Jahren mit allegorischem Holzschnitt in seiner Schrift „Praeoccupatio“ (1571)

an geowissenschaftlichen Gegenständen erst anschließend an die Zeit Agricolas bekannt. Sie sind geknüpft an den aus Basel stammenden Leonhard Thurneysser zum Thurn (1531-1596, Abb. 1), einen Anhänger des berühmten Arztes und Philosophen Paracelsus (1493-1541), der auf der Suche nach einer Druckerei für seine Schriften über Münster und Frankfurt an der Oder im Jahre 1571 nach Berlin kam. Da er es erreichte, Leibarzt des im gleichen Jahr zur Regierung gelangten brandenburgischen Kurfürsten Johann Georg (1525-1598) in Berlin zu werden und dessen Vertrauen zu gewinnen, eröffneten sich ihm sehr günstige Möglichkeiten für mannigfache Tätigkeiten, zumal er vielerlei Kenntnisse und Erfahrungen als gelernter Goldschmied, als Bergwerks- und Schmelzhüttenbetreiber in Tirol, als Alchemist sowie als Arzt und Apotheker, so zuletzt in Münster beim dortigen Bischof, mitbrachte. Im säkularisierten („Grauen“) Franziskanerkloster, von dem heute nur noch die Ruine der Klosterkirche existiert, stellte ihm der Kurfürst Räume zur Verfügung. Hier entfaltete er eine geradezu ungeheure Tätigkeit, die sich auf verschiedene Gebiete erstreckte. Er gründete die erste Berliner Buchdruckerei, die seine zahlreichen Bücher und Kalender in großen Auflagen und mit dem angestrebten hohen Niveau herausbrachte. Weiterhin betätigte er sich als Arzt bzw. als Apothekerarzt und betrieb dies auch über Ferndiagnosen und Ferntherapie, wohl trotz sei- 
ner Erfolge oft zweifelhaft. Dank der Werbung durch seine Schriften und Kalender nahm diese Tätigkeit einen großen Umfang an. Ähnlich arbeitete er auch als Astrologe und Horoskopsteller. Er war ferner Alchimist, Geldverleiher und anderes mehr. Zeitweilig beschäftigte er hierfür über 200 Personen. Auf diese Weise erwarb er sich in wenigen Jahren ein großes Vermögen.

Sein Reichtum ermöglichte ihm die Schaffung einer Bibliothek und einer Gemäldesammlung. Wohl als erster in Berlin schuf er sich auch ein Naturalienkabinett. Schon auf seinen Reisen hatte er mit Aufsammlungen begonnen. Später geht aus seinen Schriften (Thurneysser 1580 u. a.; Moehsen 1783) hervor, was er alles zusammengetragen hat: eine Sammlung von Schalen des Meeres- und Landgetiers, ein Herbar, eine Sammlung von Pflanzensamen aus aller Welt mit über 2000 Arten, von denen im Jahre 1681 schon 1689 Arten „eingeleimt" waren, etliche Tausend getrocknete tierische und menschliche Teile bzw. Zeichnungen und Modelle davon sowie schließlich eine Mineralsammlung. Über diese heißt es bei Moehsen (1783): „Seine Schreiber, die er herumschickte, sammelten für ihn aus Sachsen, Böhmen, Ungarn, Schlesien und Italien alle Mineralien, Siegelerden und seltene Steine, die zu der Zeit bekannt waren, und seine Korrespondenten in Ungarn waren ihm sonderlich dazu behilflich."

Thurneyssers glänzende Zeit in Berlin ging nach 14 Jahren $\mathrm{zu}$ Ende. Bereits zuvor hatten seine Arbeitsfähigkeit und sein Elan infolge eines Schlaganfalls nachgelassen, und er war schließlich Nachrichten aus Basel gefolgt, die ihm dort, von wo er vor Jahrzehnten fliehen mußte, günstige Aufnahme versprachen. Er fiel aber Neidern und Feinden in die Hände, die seinem Rufe durch böswillige Auslegung seiner Schriften und Tätigkeiten sehr schadeten. Auch verlor er durch Gerichtsurteil bei der Auflösung seiner unglücklichen zweiten Verheiratung sein gesamtes Vermögen. Trotz Weggang von Basel konnte er sich nicht wieder richtig emporarbeiten. Berlin hat er nicht wieder gesehen, obwohl der Kurfürst ihm gewogen geblieben war.

Auch nach dem Tode Thurneyssers setzten sich die Verleumdungen fort und ließen ihn in der Folgezeit meist als Scharlatan, betrügerischen Goldmacher, Quacksalber, Wucherer und dergleichen mehr erscheinen. Aber bereits Moehsen (1783) und vor allem genauere moderne Studien (Boerlin 1970, Morys 1982, Spitzer 1996 u. a.) erkennen ihm eine weit höhere Seriosität $\mathrm{zu}$, natürlich im Rahmen und in der Begrenztheit des Erkenntnisstandes seiner Zeit.
Was seine mineralogischen Angaben, unter anderem in seinem Werk "Pison" (Thurneysser 1572), angeht, wo er z. B. von der Gold- und Edelsteinführung in Flüssen, wie der Spree spricht, so zeichnen sich diese z. T. durch Übertreibungen aus, die aber als relativ harmlos betrachtet werden können. Thurneysser war zweifellos in manchem seiner Zeit voraus. Aber seine Vielfalt an Tätigkeiten und die Sucht, daraus größtmöglichen Gewinn zu ziehen, haben es verhindert, daß er eine Bedeutung erlangte, die ihn näher an die Agricolas gebracht hätte.

\section{Naturalien in der Kunstkammer des Berliner Schlosses}

Die nächste Spur frühen Interesses an geowissenschaftlichen Objekten führt zu den Sammlungen, die an Fürstenhöfen seit Mitte des 16. Jahrhunderts entstanden sind. Das berühmte Vorbild war die großartige Kunst- und Wunderkammer auf Schloß Ambras bei Innsbruck in Tirol, die von dem österreichischen Erzherzog Ferdinand (1520-1595) angelegt wurde. Sie enthielt in größter Vielfalt, was durch menschlichen Geist und Kunst- und Handwerksfleiß 3 geschaffen wurde und sich in Sammlungen zusammentragen ließ (Schlosser 1908) und reichte von Rüstungen und Waffen über Gemälde, Zeichnungen, Bücher, Musikinstrumente, Uhren bis zu Kleinodien und anderen kunstgewerblichen Gegenständen aus den verschiedensten Materialien. Kostbarkeit, Seltenheit und Schönheit der Objekte hatten zum Sammeln angereizt. Auch einige Naturobjekte, Silbererze und zu sogenannten Handsteinen präparierte Mineralstufen waren darunter. Die Liebhaberei des Sammelns verbreitete sich an vielen Fürstenhöfen, zumal damit auch ein Repräsentationsbedürfnis befriedigt werden konnte. Auch Bibliotheken dienten letzterem und wurden in Besichtigungen vorgewiesen. Berühmt waren ferner die Sammlungen in Dresden, die unter dem sächsischen Kurfürsten August I. (1526-1586) entstanden. Dessen ausgeprägt praktischer Sinn hatte anfangs mehr für ,eine Art Magazin mathematisch-physikalischer Instrumente" (Fischer 1939) gesorgt, in dem künstlerische Gegenstände noch in der Minderzahl waren, was sich aber in der Folgezeit änderte.

Auch am brandenburgischen Hofe wurde man von der Liebhaberei des Sammelns angesteckt. Wahrscheinlich gründete der Vorgänger von Johann Georg, der kunst- und prachtliebende Kurfürst Joachim II. (1505-1571), die Berliner 
Kunstkammer (Ledebur 1831). Nach einem Verzeichnis von 1603 waren Naturobjekte aber noch nicht vorhanden. Diese Kammer ging 1626 gänzlich in der Festung Küstrin verloren, wohin sie im 30jährigen Krieg ausgelagert worden war.

Der Neuaufbau begann unter Kurfürst Friedrich Wilhelm (1640-1688), dem „Großen Kurfürst". Er wählte dafür Räume des Apothekenflügels aus, der aus dem Rechteck des Schlosses an der Nordostecke des Berliner Schlosses herausragte. Dieses Gebäude war 1585 vom Nachfolger Johann Georgs, dem Kurfürsten Johann Friedrich (gest. 1608) erbaut worden, um darin eine Apotheke unterzubringen, die seine erste Gemahlin, Katharina, eine Schülerin Thurneyssers, auf der Moritzburg in Halle, als er dort Administrator war, eingerichtet und dann nach Berlin verbracht hatte. Über den Apothekenräumen richtete Kurfürst Friedrich Wilhelm die bis dahin nur privat genutzte Schloßbibliothek und die „Raritätenkammer" (Geyer 1936) ein, wie die Kunstkammer zunächst hieß. Selbst in der Bibliothek befanden sich Raritäten, unter anderem die von Otto v. Guericke (1602-1686) geschenkte Luftpumpe sowie die sog. Magdeburger Halbkugeln (Winter 1981), die sich jetzt in München befinden, auch sogar einige Naturalien (Döhn 1981), die erst später in die Kunstkammer kamen. Als Verwalter der Kunstkammer wurden 1663 Heinrich v. Heimbach und 1685 Christian Albrecht v. Kunckel eingesetzt, die zugleich auch Bibliothekare waren. Die Kunstkammer nannte sich zeitweilig auch Antiquitäten- und Raritätenkammer. Auch Naturalien waren vorhanden, die unter anderem aus Sendungen aus Batavia in den Jahren 1671 und 1683 stammten und unter anderem „Seegewächse“ für Grotten geliefert hatten (Ledebur 1831).

Im Jahre 1688, beim Regierungsantritt von Kurfürst Friedrich III. (1657-1713), dem späteren König Friedrich I. „in“ Preußen (ab 1701), wurde die Verwaltung dem kurfürstlichen Berg- und Münzrat Christoph Umgelter (Ungelter) übertragen. Sein erstes Verzeichnis umfaßte nach Ledebur 320 Nummern unter folgenden Positionen:

1. gedrehte Sachen, 2. Naturalien, 3. Figuren, 4. Kunststücke und Raritäten, 5. Grundrisse und Gemälde, 6. Ostindische Gewehre, 7. Mineralien und 8, mechanische Modelle.

Bis zum Tode König Friedrichs I. erhöhte sich die Anzahl auf 1500 Nummern. Aus Umgelters Zeit stammt noch ein Sammelband mit mehreren Teilkatalogen der „Churfürstlichen Raritäten Cammer", der sich in der Handschriftenabteilung der Staatsbibliothek Preußischer Kulturbesitz erhalten hat (HASTB-1; Döhn 1988). Es sind wohl Zugangskataloge für Teilgebiete, einer davon für die "Naturalia" mit den Nummern 200 bis 349. Hieraus folgen einige Beispiele:

Nr. 259: Eine runde grühne Schachtel, ... worinnen KupfferSchlacken, Blenden und Glantz Ertz.

Nr. 261: Ein Stück gediegen Silber, eines Fingers groß, so wie es auß der mine gehauen; kompt auß einer am Hartz gelegenen mine woran unsere g[nädig]ste Churfürstin participirt.

Nr. 265: Ein Stück Bern-Stein, worauff die Lupa, so Romulum und Remum gestillet hat, sich erzeiget.

Nr. 274: Ein Stücklein Stein, welcher gestaltet wie ein Affenfuß.

Nr. 275: Zwei Adlersteine, davon einer eine St. Jacobsmuschel inwendig repraesentirt.

Nr. 279: Der Stein Echinites; ist gestaltet wie ein geripter erhabener Mantel-Knopf.

Nr. 304: Das Horn vom Einhorn, welches Christianus IV. König in Dannemarken bey der hiesigen Landschafft für 18000 Thlr versetzet hat...

Die im Text der obigen Nummer 261 genannte Kurfürstin ist Sophie Charlotte (1668-1705), die Frau Friedrichs III., Tochter des Herzogs Ernst August von Braunschweig-Lüneburg, späteren Kurfürsten von Hannover, dessen Staatsgebiet bis in den Harz reichte. Die Beziehung zum Harzer Bergbau erscheint naheliegend.

Im (preußischen) Geheimen Staatsarchiv in Berlin befindet sich ein weiterer auf Umgelter zurückgehender, in weißes Pergament eingebundener Katalog mit dem Titel „Inventarium der Churfürstlich Brandenburgischen Kunst-Cammer de Anno 1694". Er ist eine Reinschrift nach dem Tode Umgelters 1693, fortgeschrieben bis 1694 (GSTAB-1). Offenbar wurde der Bestand der Kunstkammer neu geordnet und die Objekte mit anderen Nummern versehen, wohl entsprechend der Unterbringung in den mehreren Räumen und Schränken. Auch bei diesem Verzeichnis lohnt sich ein Blick in den Inhalt zur Charakterisierung des Bestandes.

Das erste Kapitel ist das „Verzeichnüß der Naturalien“. Es enthält 155 Nummern und zwar zum weitaus größten Teil zoologische Objekte, wenige botanische, etwa 25 paläontologische und wenige mineralogisch-petrographische Objekte. Der Text gestattet die Zuordnung zu diesen Kategorien nicht immer sicher, besonders bei den Versteinerungen, zumal die Reihenfolge keinerlei Systematik erkennen läßt. Es folgen hieraus einige geowissenschaftliche Objekte:

Nr. 27: Ein Podolisches Ochsen Horn, welches hier in der Marck Anno 1679 auf des Commissarii Rochau seinem Guth in der Erden gefunden, und darin zu Stein worden.

Nr. 66: Vier Tropfsteine, seynd im alten Inventario Eiszapffen genennet worden, so im Sauerländischen felsichten $\mathrm{Ge}-$ birge zu Stein worden.

Nr. 84: Zwei Stücke Bernstein, ... in Pommern gefunden. Nr. 86: Ein Donnerkeil, so Seiner Churfürstlichen Durchlaucht von Herrn Martitio davor gegeben worden. 
Nr. 101: Ein Stein in Gestalt eines Affenfuß.

Nr. 122: Ein Stück Cristall, repraesentirt inwendig Nadeln. Nr. 127: Ein Stück polirt Agat Stein.

Nr. 129: 45 Stück gefärbte Marmor-Stein und Gipß Proben.

Nr. 144: Ein gar groß und zwey kleine ungearbeitete Jaspis, so Seine Churfürstl. Durchlt. von Sachsen Sr. Churfürstl. Durchlt. von Brandenburg verehrt.

Nr. 155: Acht Stück Bernstein.

Das zweite Kapitel des Kataloges von 1694 ist das „Verzeichnüß der Mineralien“, das nur Metalle und Erze enthält. Gegenüber der Unordnung des Naturalienbestandes herrscht hier eine systematische Reihung, die offenbar von der Alchimie vorgegeben wurde. Dieses Kapitel wird vollständig abgedruckt:

Goldt Ertzt

Nr. 1: Eine Stuffe gewachsen Gold in weißem Quartz. Ist aus Indien von der Insel Sumatra kommen, wiegt 5 Pfd., 7 Loth, $1 / 4$ Quint.

Nr. 2: Chinesische Goldarbeit und Goldkörner, wiegen zusammen 6 Loth.

Silber Ertzt

Nr. 1: 13 Stüfflein gewachsen Silber in weißem Quartz, seyend aus Norwegen. Wiegen 2 Pfd., 14 1/2 Loth.

Nr. 2: Herr Cammerdiener Stochius hat in die Churfürstl. Kunstkammer geschenckt: Eine rar gewachsen Silber Stuffen, wiegt 1 Pfd.

Eißen

Nr, 1: Eine große Eisen Blume

Nr. 2: Ein Stück Eisen Blüt.

Nr. 3: 23 Eisen Stuffen.

Kupffer Ertzt

Nr. 1: Zwei Huff Eisen, welche in Kupffer verwandelt sind in einem Brunnen der in Ungarn bey Neusohl ... seyn soll. Davon eins zerbrochen

Nr. 2: 24 Kupffer Stuffen.

Zinn

Nr. 1: 16 Zinn Stuffen. Ein Zinn graupe.

Mercurialische, Arsenicalische, Sulverische und dergl. Steine

Nr. 1: 71 Stuffen Mineralien, Ertze und Gesteine.

Nr. 2: 33 geschmelzte Sachen, Kies und Metall.

In allen übrigen Kapiteln des Verzeichnisses von 1694, in denen die Namen von Naturalien und Mineralien genannt werden, handelt es sich um solche Objekte, die bereits zu Schmuckgegenständen oder dergleichen verarbeitet sind. Sogar im dritten Kapitel, im „Verzeichnüß allerhandt Edelgesteine", das dies nicht erwarten läßt, handelt es sich um geschliffene, skulpturierte und zum Teil gefaßte Schmucksteine, wie folgendes Beispiel zeigt:

Nr. 7: Ein Jaspis in Form eines Herzens, repraesentieret auf einer Seiten einen alten Mannes Kopf, ist in Gold eingefasset.

In den 11 Nummern dieses Kapitels treten folgende Mineralnamen (mit der Schreibweise des Verzeichnisses) auf:

Granat, Crystall, Chrysolit, Jaspis, Lapislazuli, Achat, bömischer-Diamant, Hyacinth, Ametyst, Carniol und Lapis Nephriticus.

Während es über die einzelnen Zugänge des Bestandes durch Angaben im Verzeichnis manche Kenntnisse gibt, sind Nachrichten über Abgänge sehr selten. Ein solcher Fall ist die Abgabe von einigen Dubletten zoologischer und mineralogischer Objekte an das (noch heute existierende) Naturalienkabinett des bekannten Pfarrers und Pädagogen August Hermann Francke (1673-1727) in Halle, der den Kurfürsten im Jahre 1698 um einige Stücke gebeten hatte (Ledebur 1831). Er wollte die Objekte als Anschauungsmaterial im Schulunterricht verwenden, ein anderes, zeitgemäßes Motiv für die Benutzung naturkundlicher Sammlungsgegenstände, das in den fürstlichen Naturalienkammern nicht im Vordergrund stand. Francke erhielt die erbetenen Stücke, wie Eintragungen im nächstfolgenden Katalog zeigen.

Der nächste Katalog der Kunstkammer stammt aus dem Jahre 1716 und ist eine fortgeschriebene Abschrift des Kataloges von 1694. Er befindet sich im Archiv der Berlin-Brandenburgischen Akademie der Wissenschaften (ABBA-1). Die Anzahl der Naturalien ist mit 406 angegeben. In der Art der Bestandsordnung und Darstellung gibt es keine Änderung, auch die Nummern der Objekte blieben die gleichen. Neue Objekte wurden am Schluß zugefügt und manche Texte zu den Stücken wurden etwas gekürzt.

Das gleiche trifft auf den Katalog von 1756 zu, der 466 Naturalien verzeichnet. Auskunft über diesen Katalog, der nicht eingesehen werden konnte, gibt ein Artikel über die Geschichte der Sammlungen des Geologisch-Paläontologischen Institutes der Humboldt-Universität (Dietrich 1960). Danach stammt der Katalog von Wilhelm Stosch, der nach Degering (1922) zunächst als Adjunkt der Königlichen Bibliothek tätig war und erst im Jahre 1765 die volle Anstellung bekam. Zu diesem Zeitpunkt erfolgte die Trennung der Kunstkammer von der Bibliothek und Stosch erhielt die Aufsicht über die Kunstkammer. Dietrich war der Ansicht, daß dieser Katalog ,wohl der älteste Katalog einer Berliner Naturaliensammlung" sei. Er zitiert daraus insgesamt $7 \mathrm{Ob}$ jekte, von denen die ersten vier in Nummer und Text mit den Nummern 27, 86, 101 und 129 des Kataloges von 1694 (siehe dort) übereinstimmen, und folgende drei Objekte neu sind:

Nr. 171: Ein Krötenstein, Bufonit oder Schlangenauge, auch Batrachit oder Crapaudine genannt (Dietrichs Anmerkung: Es sind Gaumenzähne von ... Fischen).

Nr. 186: Ein Stück unicornu fossile.

Nr. 328: Eine Glossopetra so Markgraf Albrecht dem König verehret.

Also auch bei den neueren Eintragungen wurden altertümliche Bezeichnungen verwendet. Dietrich begleitet dies mit der Bemerkung: „Es sind die üblichen Curiosa der Zeit." Einflüsse Carl v. Linnés (1707-1778) sind nicht zu spüren. 
Im Jahre 1769 erließ König Friedrich II. (1712-1786) die Weisung an das Bergwerksund Hütten-Departement seiner Regierung, das Naturalien-Kabinett der Kunstkammer „mit den künftig aufzufindenden Naturalien und den demselben fehlenden Erzstufen zu versehen" (Ledebur 1831). Friedrich II. hatte inzwischen die drei Schlesischen Kriege geführt und dadurch war Preußen im Jahre 1763 in den Besitz Schlesiens mit seinen Bodenschätzen gelangt. Der Weisung von 1769 folgte ein Jahr später die Gründung der Berliner Bergakademie, worauf weiter unten eingegangen wird.

Das weitere Schicksal der geowissenschaftlichen Bestände der Kunstkammer nach 1770 kann nur noch stark verkürzt im folgenden angedeutet werden.

Nachdem die Kunstkammer im Jahre 1794 einen neuen Verwalter, den Prediger der französischen Gemeinde Jean Henry (1761-1831) erhalten hatte (GSTAB-2) und der Akademie der Wissenschaften im Jahre 1798 mit letzterem unterstellt worden war, ordnete König Friedrich Wilhelm III. (1770-1840) im Jahre 1805 die Abgabe sämtlicher Mineralien an die inzwischen sehr gut entwickelte Sammlung der Bergakademie, das Königliche Mineralienkabinett, an (ABBA-2; Reichl 1930), zumal in der Kunstkammer kein wissenschaftlicher Gebrauch davon gemacht wurde (GSTAB-3). Die Überführung unterblieb aber für einige Jahre aus mehreren Gründen, so wegen Überfüllung, wegen Protesten von Henry (GSTAB-2) und wegen der Lähmung des gesamten öffentlichen Lebens infolge des Einfalls der Franzosen im Jahre 1806 und der Besetzung Berlins. Im Jahre 1809 erfolgte schließlich die Abgabe an das Königliche Mineralienkabinett (ABBA-3), was dessen Bestände nach Umfang und Wert nur in recht bescheidenem Maße vermehrte. Als dann im Jahre 1810 die Vorstellungen Wilhelm von Humboldts zur Errichtung der Berliner Universität realisiert wurden, gelangte das Königliche Mineralienkabinett als Mineralogisches Museum in den Besitz der Universität.

Von den übrigen naturkundlichen Objekten der Kunstkammer und der Akademie der Wissenschaften wissen wir, daß die zoologischen Objekte 1810/11 ebenfalls der Universität übereignet und zum Aufbau von deren Zoologischem Museum verwendet wurden.

\section{Private Sammlungen in Berlin}

Neben den fürstlichen Kunst- und Naturalienkammern entstanden etwa von der Zeit Agricolas an auch Naturaliensammlungen privater Personen. In Italien und Holland geschah dies sogar vereinzelt schon früher. Besonders waren es Apotheker, deren Materiallager ja zugleich Naturaliensammlungen darstellten oder sich leicht $\mathrm{zu}$ solchen Kammern herrichten ließen, die man zu Führungen und Besichtigungen öffnen konnte (Jahn 1979). Verzögerungen dieser Entwicklung gab es in Ländern, die besonders stark vom wirtschaftlichen Niedergang infolge des Dreißigjährigen Krieges betroffen waren. Aber auch dort zeigten sich im ausgehenden 17. Jahrhundert die Spuren des Auflebens, zum Teil in bemerkenswertem Maße. So schuf August Hermann Francke in Halle für den Unterricht sein Naturalienkabinett. In Leipzig entstand in der Apotheke von Heinrich Linck (gest. 1717) ein Naturalienkabinett, das von Sohn und Enkel weiter ausgebaut wurde (Fischer 1939) und das in Waldenburg erhalten geblieben ist. Als drittes Beispiel sei noch die Mineraliensammlung des Freiberger Bergrates Johann Friedrich Henkel (Henckel) (1679-1744) genannt, der sich eingehend mit Mineralogie beschäftigte und diese durch seine „Kies-Historie“ (Henkel 1725) und andere Schriften stark gefördert hat.

Auch in Berlin erwachte das Interesse an der Beschäftigung mit Naturobjekten und es entstanden vom Ende des 17. Jahrhunderts an die ersten Naturaliensammlungen von Bürgern. Es zeigten sich auch Spezialisierungen, indem bestimmte Objektgruppen bevorzugt wurden. Folgende Liste gibt eine Übersicht solcher Berliner Bürger, die Mineralien einschließlich Versteinerungen ausschließlich oder teilweise sammelten. Diese Zusammenstellung reicht bis etwa zur Gründungszeit der Berliner Bergakademie, was jedoch eine willkürliche Grenze darstellt, da sich das Sammeln weiter kontinuierlich entwickelte.

Wie die Übersicht zeigt, reichen die Berufe der Mineralsammler über einen großen Spielraum. Neben einem Bergrat sind ein hoher Bergbeamter, ein Prediger, ein Botaniker, zwei Apotheker, ein Chemiker und fünf Ärzte vertreten, wobei sich einige Ärzte ebenso wie die Apotheker auch intensiv als Chemiker betätigt haben.

\footnotetext{
1 Mineralien = Sammelbegriff im Sprachgebrauch der Zeit für geowissenschaftliche Objekte.

2 Slg. = Sammlung
} 


\section{Sammler von Mineralien ${ }^{1}$ in Berlin}

Stahl, Georg Ernst (1660-1734), Arzt und Chemiker, Leibarzt Friedrich Wilhelms I., Begründer der PhlogistonTheorie; Slg. ${ }^{2}$ von Mineralien, Katalog 1773 (Wilson 1994).

Spener, Christian Maximilian (1678-1714), Arzt, Professor der Anatomie am Theatrum Anatomicum; Slg. von Naturalien allgemein, Katalog 1718 (Wilson 1994).

Neumann, Kaspar (1683-1737), Apotheker und Chemiker, Professor der praktischen Chemie beim Collegium medico-chirurgicum; Slg. von Mineralien, Katalog (Neumann 1767).

Eller, Johann Theodor (1689-1760), Arzt und Chemiker, Leibarzt Friedrichs II., Direktor des Collegium medicochirurgicum; Slg. von Mineralien, Katalog 1723 (Neickelius 1727), nochmaliger Katalog (Eller 1761)

Pott, Johann Heinrich (1692-1777), Arzt und Chemiker, Professor der theoretischen und (ab 1737) der praktischen Chemie beim Collegium medico-chirurgicum, schließt 1746 auf eine besondere Erde in den verglasbaren Steinen $\left(\mathrm{SiO}_{2}\right)$; Slg. von Mineralien (Pott 1746/54).

Marggraf, Andreas Sigismund (1709-1782), Apotheker und Chemiker, Chemiker der Akademie der Wissenschaften, entdeckt 1754 die "Alaun“- bzw. „Thonerde“ $\left(\mathrm{Al}_{2} \mathrm{O}_{3}\right)$; Slg. von Mineralien, Verkaufsangebot (Bernoulli 1782).

Gleditsch, Johann Gottlieb (1714-1786), Botaniker, Lehrer am Collegium anatomico-chirurgicum, Direktor des Botanischen Gartens; Slg. von Herbar, Versteinerungen, Konchylien (Sander 1784, Nicolai 1786).

Lehmann, Johann Gottlob (1719-1767), Bergrat, schrieb mineralogisch-geologisch-bergbaukundliche Werke und Lehrbücher, hielt private Vorlesungen (v. Freyberg 1955), ging 1761 nach St. Petersburg; Slg. von Mineralien (Lehmann 1758).

Redern, Sigismund Ehrenreich Graf von (1720-1789), Oberhofmarschall; Slg. von Mineratien (Wilson 1994).

Woltersdorf, Johann Lukas (1721-1772), Prediger an Berliner Kirchen; Slg. von Mineralien (Woltersdorf 1748), Katalog 1772 (Wilson 1994).

Bloch, Marcus Eliesar (1723-1799), praktischer Arzt und Zoologe, Arbeiten über Fische; Slg. von Naturalien, besonders Fischen, auch Mineralien (Sander 1784).

Kurella, Ernst Gottfried (1725-1799), praktischer Arzt; Slg. von Mineralien, Konchylien (Sander 1784, Nicolai 1786).

Nur eine dieser Sammlungen umfaßte den gesamten Umfang der Naturalien in ähnlicher Weise, wie es in der Kunst- und Naturalien-Kammer des königlichen Schlosses der Fall war. Es ist die Sammlung des Arztes Christian Maximilian Spener, eines Mitgliedes der bekannten Berliner Theologen- und Verlegerfamilie. Ihm haben offenbar reiche Mittel für ihren Aufbau zur Verfügung gestanden. Im Jahre 1692 wurde diese Sammlung vom Verwalter der Kunst-Kammer des Berliner Schlosses Christoph Umgelter begutachtet, wohl wegen eines möglichen Ankaufs von Stücken. Umgelter notierte über die Bestände: „Von den Metall und Mineralischen Ertzen, Gesteinen und in Stein verwandelten Sachen, Erden, Mineralien und Quartzen etzl. sind auch ohngefähr nur dreißigster Teil dabey, welche bei der Churfürstl. Cammer nicht vorhanden." (HASTB-2). Erst nach Speners Tod wurde der größte Teil der Sammlung Speners von der Aka- demie der Wissenschaften gekauft und fand eine repräsentative Aufstellung in Räumen der Akademie, dem Observatorium.

Alle übrigen Sammlungen zeigten eine mehr oder weniger starke Einengung des Sammelgebietes, was finanziell bedingt sein kann, aber wohl eher den speziellen Interessen der Sammler entsprach. Bei den chemisch tätigen Sammlern lag dies nahe, da die damalige Chemie vorwiegend eine Mineralchemie war. Der bedeutendste Chemiker unter den Ärzten war Georg Ernst Stahl. Er ist für die Entwicklung der Chemie von erheblicher Bedeutung, da er die Phlogistontheorie, die bis gegen Ende des 18. Jahrhunderts herrschend war, aufgestellt hat. Auch seine Schüler Kaspar Neumann, Johann Heinrich Pott und Andreas Sigismund Marggraf, zugleich Schüler Henkels, waren bedeutende Chemiker und daneben Mineraliensammler. Besonders traf dies auf den letzteren zu. Und dies wurde im Verkaufsangebot ausgenutzt, das ein Akademie-Kollege, der Astronom Johann Bernoulli (1744-1807) im Jahre 1782 erscheinen ließ, um die Sammlung attraktiv zu machen. Dort heißt es: „Eine von einem so großen Chymisten angelegte Sammlung kann, wie leicht zu erachten, nicht anders als vorzüglich seyn", noch dazu, weil schon zu Lebzeiten Marggrafs 200 Louisdor dafür geboten worden waren.

Von einem weiteren Arzt und Chemiker, Johann Theodor Eller, ist ein sehr detaillierter Katalog überliefert, der nach seinem Tode erschienen ist. Sehr viele Stücke dieser Sammlung sind einzeln genau beschrieben, so zum Beispiel allein unter der Kategorie "Silber-Ertzte" 378 Stücke. Jedoch ist es, wie in dem als Vorwort vorausgeschickten „Avertissement" des Kataloges von dem anonymen Bearbeiter hingewiesen wird, ,zu beklagen, daß sich kein ordentlicher catalogus von dieser schönen Sammlung (hat) finden wollen. Eben daher rührt es, daß man bey denen wenigsten Piècen den locum natalem (hat) anführen können." Aber den meisten Sammlern machte das hier beklagte Fehlen des Fundortes nichts aus. Neben den Mineralen im heutigen Sinn finden sich in der Sammlung auch mehrere Kategorien von Versteinerungen. Diese sind vielfach ebenfalls einzeln beschrieben, aber auch gebündelt, wie zum Beispiel: „mehrere hundert versteinerte Muscheln und Schnecken theils in matrice, theils extra matricem". Zum Schluß folgt noch eine große Sammlung rezentes zoologischer Objekte „Muscheln, Corallophytis und andere Marinis" (Eller 1761). Der Verbleit der Sammlung ist unbekannt. 
Die Sammlung des Predigers Johann Lukas Woltersdorf ist dadurch bemerkenswert, daß sich ihr Besitzer bemüht hat, ein Ordnungssystem zu schaffen, das er im Jahre 1748 gesondert publizierte. Es sollte dazu dienen, ,damit nicht ein uneingerichteter Hauffe von Bergarten mir zur Last fallen und der, von der Sammlung gehofte Nutzen ausbleiben möchte. $\mathrm{Zu}$ dem Ende zog ich, so viel möglich, die Schriftsteller dieser Wissenschaft zu Rathe." Er nannte dann folgende „zu ihren Zeiten berühmten" Schriftsteller: den Züricher Arzt Conrad Gesner (1516-1565), den Leibarzt des Kaisers Rudolf II., Boethius De Boot (um 1600) in Wien, den Londoner Medizinprofessor John Woodward (1665-1728) und den Züricher Naturforscher Johann Jacob Scheuchzer (1672-1733). Woltersdorf war aber enttäuscht, da er bei ihnen nicht die Hilfe fand, die er suchte. Die beiden letzteren sind übrigens sehr wichtig geworden, da sie die zuvor oft als Spiele der Natur angesehenen Tier- und Pflanzenreste als Versteinerungen erkannten. Selbst Woltersdorfs Versuch mit dem System von Carl v. Linné (1707-1778), das er nach 1740 kennen lernte, brachte ihm nicht das gewünschte Ergebnis, so daß er sich eine Einteilung auf der Grundlage der "Mischung", also nach der chemischen Zusammensetzung wie folgt zurechtlegte (in seiner Orthographie):

$\begin{array}{ll}\text { I Erden } & \text { 1. Thonartige Erden (Ton, Humus) } \\ \text { II Steine } & \begin{array}{l}\text { 2. Kalkartige Erden (Kreide, Mergel) } \\ \text { 1. Glasartige Steine } \\ \text { (u. a. Edelsteine, Quartz, Flußspat) }\end{array} \\ & \begin{array}{l}\text { 2. Thonartige Steine } \\ \text { (Asbest, Talck, Blende, Schiefer) }\end{array} \\ \text { 3. Gypsartige Steine } \\ \text { (Gypstein, Alabaster, Gypsspath) } \\ \text { 4. Kalkartige Steine }\end{array}$

III Saltze

IV Bitumina

V Semimetalle

VI Metalle

Petrefakta

Der letzte Sammler, zu dem hier noch gesondert Stellung genommen wird, ist Johann Gottlob Lehmann (Abb. 2). Er wurde in Langenhennersdorf bei Pirna geboren und war ursprünglich Arzt, hatte sich aber in Dresden bald bergbaulichen und mineralogischen Studien zugewandt. Seit 1750 in Berlin, entwickelte er sich zu einem bedeutenden Naturforscher, der sich sehr vielseitig betätigte, so daß er im Titel seiner Biographie (v. Freyberg 1955) als Arzt, Chemiker, Metallurg, Bergmann, Mineraloge und grundlegender Geologe bezeichnet wird. Seine Mineraliensammlung hat er vornehmlich bei seinen Arbeiten in Bergwerken und im Gelände

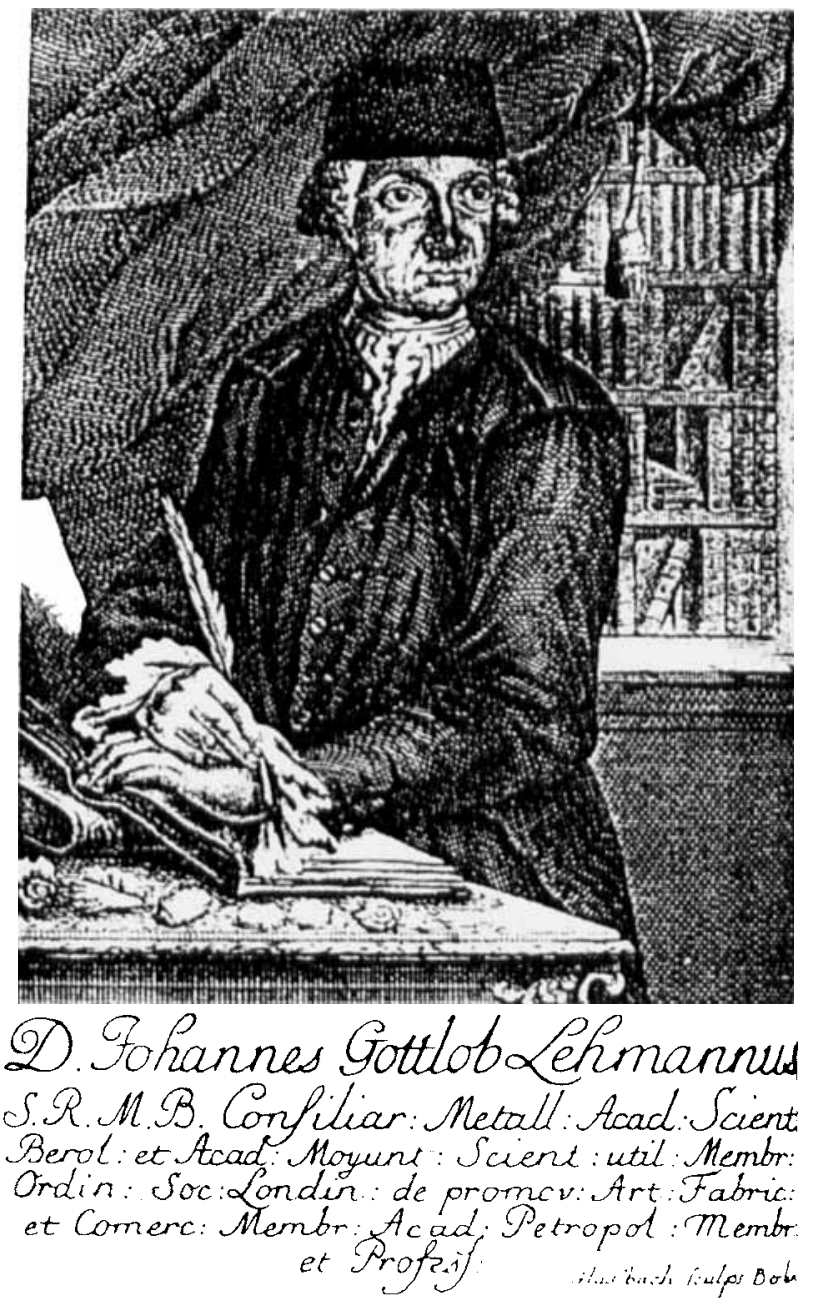

Abb. 2. Johann Gottlob Lehmann (1719-1767). Porträt im Alter von 41 Jahren aus seinen ,physikalischchymischen Schriften“ (1761)

zusammengetragen. Er hatte eine merklich andere Einstellung zum Sammeln als zu seiner Zeit üblich. Man kann dies aus seinen Lehrbüchern und anderen Schriften entnehmen, die er in groBer Zahl über seine geowissenschaftlichen Arbeiten im Gelände und im Laboratorium herausgebracht hat. Er legte vor allem solche Stücke in seine Sammlung, von denen er es für notwendig hielt, sie so zu haben, ,wie sie gefunden werden, da denn öfters eine schlecht aussehende Pièce instructivischer ist, als die kostbarste und reichste Schaustuffe" (Lehmann 1758). Diese und weitere Äußerungen Lehmanns zum Sammeln hat B. v. Freyberg (1955) zusammengetragen, hier noch ein Beispiel (Lehmann 1761a):

„Es ist wohl nichts abgeschmackter, als wenn ein Sammler natürlicher Seltenheiten, solche bloß zu dem Ende sammelt, damit er sie haben möge, ohne sich weiter um eine nähere und genauere Erkenntniß derselben zu bekümmern, ... Gewiß diese Leute verdienen unter denen Naturforschern weiter keinen andern Rang, als bloß den Titel schöner Raritätenkrämer.“ 
Am höchsten stellte Lehmann also den wissenschaftlichen Wert der Sammlungsstücke, was, wie v. Freyberg bemerkt, für die ästhetischen Grundsätze und Ziele der damaligen Sammler unerhört war.

\section{Zur Gründung der Berliner Bergakademie}

Der zuletzt als Mineralsammler dargestellte Johann Gottlob Lehmann leitet unmittelbar zur Betrachtung der Entstehung der Berliner Bergakademie über, da Lehmanns Tätigkeit als Bergrat das Bergwesen der damaligen Zeit in PreuBen charakterisiert. Trotz des vom König verliehenen Titels als Bergrat hatte Lehmann keine feste Anstellung, auch nicht als Mitglied der Berliner Akademie der Wissenschaften, wozu er im Jahre 1754 vom König ebenfalls ernannt worden war. In der Akademie hatte er durch die Mitgliedschaft die ungewisse Aussicht auf eine besoldete Stelle im Falle des Ausscheidens eines Stelleninhabers erhalten. Allerdings wurden ihm des öfteren „Commissionen“, zeitlich begrenzte Arbeitsaufträge, zu bestimmten Untersuchungsoder Erkundungsaufgaben erteilt, zunächst in kleinerem Umfang. Von 1752 an war er wiederholt für jeweils mehrere Monate ,auf allerhöchsten Befehl" in den relativ wenigen preußischen Bergbaugebieten unterwegs. Abgebaut wurden besonders Raseneisenerze, Alaunerden, Torfe und Kohlen, auch gab es Salzgewinnung in Salinen. Eine Ausnahme machte der Harz, jedoch besaß Preußen nur kleine Teile des bergbaulich interessanten, aber in staatlicher Hinsicht stark zerstückelten Harzes. Sie lagen am nördlichen Harzrand bei Wernigerode, am Südharz in der Grafschaft Hohnstein bei Nordhausen und in der Grafschaft Mansfeld im und am Ostharz. Dorthin wurde Lehmann mehrmals gesandt. Anschließend bekam er den Auftrag, das vorläufig eroberte Schlesien zu bereisen. Diese bergbaulich wertvolle Provinz hatte Friedrich II. in zwei Schlesischen Kriegen (1740/2 und 1744/5) mühsam erkämpft, jedoch war der Besitz wegen der politischen Lage in Europa noch nicht endgültig gesichert. Lehmann besuchte Schlesien 1755 und im Frühjahr 1756, d. h. vor Ausbruch des Siebenjährigen Krieges, auch wieder ,auf allerhöchsten Kgl. Befehl".

Auf seinen Reisen in Preußen achtete Lehmann bei den bergbaulichen Befahrungen überall auch auf die auftretenden Gesteine, auf deren Schichtenfolge und auf die Lagerungsverhältnisse. Dies verarbeitete er in seinem Buch, das er "Versuch einer Geschichte der Flözgebürge“ (Lehmann 1756) nannte. Es ist ein grundlegendes Werk, das die stratigraphische und erdgeschichtliche Erforschung der Erdkruste einleitete.

Bei den Befahrungen der Berg- und Hüttenwerke erhielt Lehmann den Eindruck, daß es mit dem fachlichen Wissen der Bergbeamten oft nicht zum Besten bestellt war, was ihn anregte, in Berlin Vorlesungen zu halten. Er schrieb auch schon sehr früh ein Lehrbuch, eine Einführung für Anfänger im Bergwesen (Lehmann 1751). Später folgten sein Lehrbuch der Mineralogie, das sehr gefragt war und eine zweite Auflage erlebte (Lehmann 1758, 1760) sowie seine Probierkunst (Lehmann 1761).

Von Mitte 1756 blieben „Commissionen“ für Lehmann aus. $\mathrm{Zu}$ diesem Zeitpunkt hatte Friedrich II. mit dem Einfall in Sachsen und Böhmen den dritten Schlesischen Krieg, der schließlich 7 Jahre dauerte, begonnen, um den Besitz Schlesiens zu sichern. Da nun Gelder für die Förderung des Bergwesens ausblieben, kam Lehmann in eine schwierige Lage. Durch Gratifikationen von der Akademie für geleistete chemische Untersuchungen wurde er nur notdürftig unterstützt. Der Krieg verlief wechselhaft. Im Jahre 1759 war er nach der Niederlage in der Schlacht von Kunersdorf für Preußen fast verloren, aber erneute Anstrengungen Preußens glichen dies wieder aus. Ein Ende war nicht abzusehen und große Ungewißheit herrschte.

In dieser Situation erhielt Lehmann den Ruf nach St. Petersburg als Professor für Mineralogie an die dortige Akademie der Wissenschaften. Er nahm den Ruf an, was in seiner Lage nur zu verständlich ist, und verließ Berlin Mitte 1761. In St. Petersburg konnte er ohne finanzielle Sorgen arbeiten, was er auch mit großem Elan und Erfolg tat (Barchatova \& Raskin 1976). Übrigens machte Lehmann im russischen Bergwesen die gleiche Erfahrung wie in Preußen über die unzulängliche Ausbildung des Personals und trat am 27. 4. 1766 mit einer Denkschrift auf, in der er Vorschläge zur Verbesserung machte (Raskin 1974; für den Hinweis auf diese Publikation sei Herrn Dr. Peter Krüger auch an dieser Stelle herzlich gedankt). Lehmanns Schrift trägt den Titel: „Patriotische Gedanken, wie dem Verfall derer Bergwerke durch Anlegung eines Berg-Cadetten-Corps zu helfen sey." Er wurde dadurch $\mathrm{zu}$ einem der Gründer der Bergakademie in St. Petersburg, die im Jahre 1773 eröfnet wurde. Lehmann erlebte dies aber nicht. Er starb bereits am 11. Januar 1767 an einem Gallenleiden 
oder, wie auch überliefert ist, an einer Arsenikvergiftung durch einen Laborunfall.

Bereits vor Lehmanns Weggang war im Jahre 1758 ein Bewerber um die Verwendung im preuBischen Staatsdienst in Berlin aufgetreten, der Ansprüche auf eine höhere Position geltend machte und wohl auch wegen seines Adelstitels machen konnte, der Staats- und Wirtschaftswissenschaftler Bergrat Johann Heinrich Gottlob v. Justi (1720-1771) (Allg. Deutsche Biographie, Bd. 14). Sein Antrag hatte Erfolg, wenn auch mit Vertröstung auf die Zeit nach dem Kriegsende. Jedoch erhielt v. Justi vom König sogleich eine Art Wartegeld in Gestalt eines kleinen, aber "verheerten" Landgutes bei Berlin. Er betätigte sich nun zunächst als höchst produktiver kameralistischer Schriftsteller. Auch zuvor war er als solcher aufgetreten, hatte aber dabei ein recht unstetes Leben geführt. So mußte er seine erste hervorragende Position als Professor der Kameralwissenschaft und als Finanz- und Bergrat in Wien aufgeben, als seine Silbergewinnung aus Kalken von Annaberg in Niederösterreich 1753 scheiterte. Weitere, zunächst Erfolg versprechende Versuche, Fuß zu fassen, unternahm er in Göttingen, Hamburg und Kopenhagen. Auch eine Silbermünzenaufarbeitung, die er 1763 in Hamburg während der Berliner Wartezeit einrichtete, gab er nach sehr gutem Anfangserfolg auf. Ein erneuter Antrag vom 14.2.1762 auf Einstellung als Geheimer Finanzrat (GSTAB-6) hatte keinen Erfolg. Im März 1766 schließlich wurde er von Friedrich II. zum Berghauptmann und Ober-Aufseher der Glas- und Stahlfabriken ernannt und erhielt wegen seiner Sehschwäche, die schnell zur Erblindung führte, einen Beamten als persönlichen Helfer. Aber schon 1768 nahm seine Tätigkeit ein Ende, da er wegen des Kassendefizites von 46000 Thlr., nach eigener Angabe 2700 Thlr. (GSTAB-7), seines Postens enthoben und auf die Festung Küstrin gebracht wurde. Dort starb er im Juli 1771.

Zum Auftreten v. Justis in Preußen sei noch nachgetragen, daß Lehmann mit ihm Berührung gehabt hat. Lehmann äußerte sich darüber gegenüber seinem Briefpartner Johann Ambrosius Beurer (1716-1754), einem in Berlin ausgebildeten Apotheker in Nürnberg am 16. 4.1754 wie folgt (v. Freyberg 1955):

„Muß Thnen berichten, daß der so berühmte und glückliche Entdecker derer reichen Kalksteine in Niederösterreich $\mathrm{zu}$ St. Annaberg, der H. von Justi sich in unseres Königs Landen aufhält, nehmlich in der Stadt Mannsfeld. Es ist hiesiges Ortes fast unbegreifl. warum dieser Mann, nachdem er doch seine Religion changirt, und eine Bedienung von $5000,-\mathrm{R}$. gehabt, sein reiches Bergwerk, seinen ansehnl. Gehalt und alles verlassen, und NB. in schlechten Umständen aus Wien gegangen, da er so gar selbst saget, man habe noch biß dato seine Meubles, Cabinet pp. in Wien mit Arrest belegt. ... Hier formirt der Mann Projecte, welche sehr windig sind, und mir zur Untersuchung anvertraut sind. Was mich gegen denselben noch soupconneuser [argwöhnischer] macht, ist $\mathrm{da}$. er mir von seinen Ertzten, aus St. Annaberg einige kleine Proben gegeben, mit dem Bedeuten, es hätten solche, die beste Art 20 Mark Silber, die geringste aber 8 Mark; bey angestellter Probe aber in der besten nur 2 Mark und in der andern 1 Mark 13 Loth gefunden."

Spätere Nachrichten zum Verhältnis Lehmanns zu v. Justi sind nicht bekannt geworden, auch nicht, ob es einen Einfluß v. Justis auf Lehmanns Weggang von Berlin gegeben hat.

Bei der Einsetzung v. Justis war Friedrich II. offenbar entschlossen, das Bergwesen Preußens organisatorisch zu stärken und eine einheitliche Leitung zu schaffen. Der Titel Berghauptmann deutet darauf hin, daß nicht nur die Glas- und Stahlfabriken, d. h. das Hüttenwesen, reformiert werden sollte. Jedenfalls wurden schon im Jahre 1766 erste Veränderungen auf dem Gebiet der Bergordnungen und Berggesetzgebung eingeführt (Daber 1970). In der Regierung Preußens, das heißt im General-Direktorium (genauer: General-Ober-Finanz-, Krieges- und Domänen-Direktorium), wurde dann im Jahre 1768 eine gesonderte Abteilung geschaffen, das Bergwerksund Hütten-Departement. Es scheint auch schon der Gedanke der Schaffung einer besonderen Lehranstalt entstanden zu sein, was nahelag, da eine solche bereits 1765 in Sachsen errichtet worden war.

Im Jahr 1768 trat der in Berlin seit einigen Jahren praktizierende Arzt Carl Abraham Gerhard (1738-1821, Abb. 3) an König Friedrich II. mit dem Antrag heran, ,als Mitglied in die Königliche Preußische Akademie der Wissenschaften aufgenommen zu werden, um 'würdig seinen König zu vertreten', wenn er in Verhandlungen bezüglich der beabsichtigten Gründung einer Bergakademie treten muß" (Bielefeldt 1980).

Gerhard stammte aus einer schlesischen $\mathrm{Pa}$ storenfamilie. Er studierte in Frankfurt an der Oder Medizin, befaßte sich aber dabei auch mit Mineralien und schloß das Medizinstudium 1760 mit einer Dissertation über schlesische und böhmische Granate ab. In den Folgejahren trat Gerhard mit „physiko-medizinischen“ Schriften auf, die, wie seine Übersetzung der französischen $\mathrm{Pu}$ blikationen J. T. Ellers auch mineralogische Artikel enthielten.

Der Ausfall des Berghauptmanns v. Justi scheint König Friedrich II. bewogen zu haben, den Antrag Gerhards in Erwägung zu ziehen. Darauf deutet hin, daß das General-Direktorium 


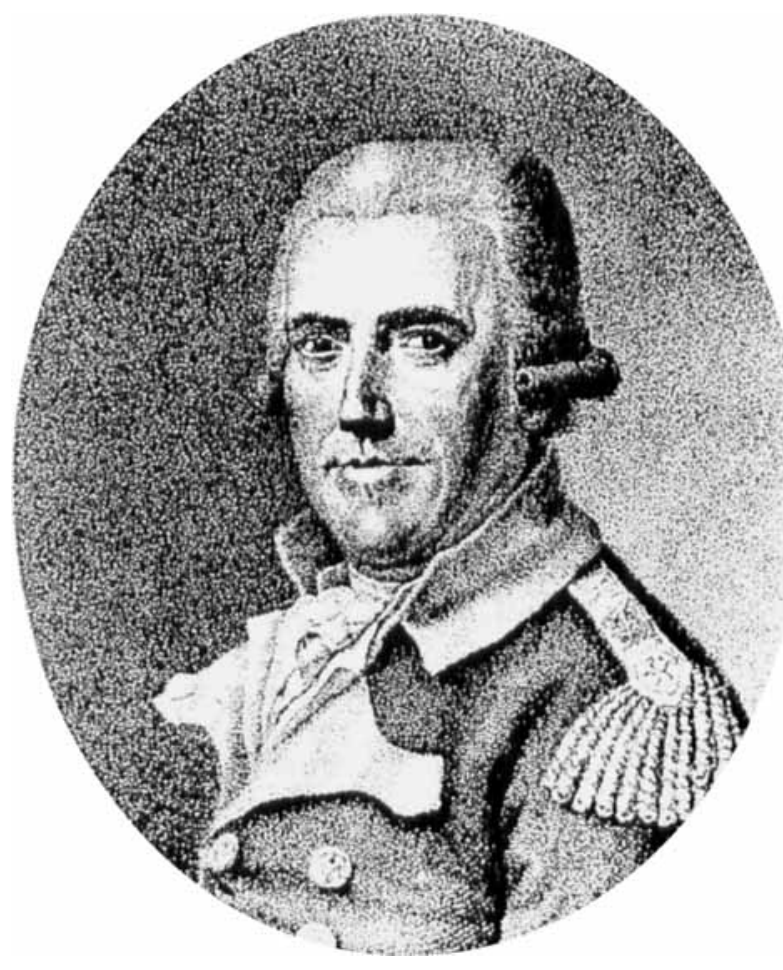

Abb. 3. Carl Abraham Gerhard (1738-1821). Porträt als Königlich Preußischer geheimer Ober-Finanz-, Krieges- und Domänen-Rat, zu dem er im Jahre 1786 ernannt wurde. Original im Märkischen Museum, Berlin

die Stelle eines Bergrats für Gerhard bewilligte, was am 21.4.1768 in der Sitzung der Akademie der Wissenschaften mitgeteilt wurde (ABBA-4). Friedrich II. bildete dann eine Immediat-Kommission, bestehend aus dem Geheimen Finanz-, Krieges- und Domänen-Rat Heinrich Wilhelm Reichard und Bergrat Gerhard. Ersterer war der höchste Beamte im Bergwerks- und Hütten-Departement unter dem Minister und hatte zuvor der Jurisdiktions-Kommission des General-Direktoriums angehört. Die Immediat-Kommission bekam den Auftrag, das schlesische Bergwesen zu untersuchen und Maßnahmen zu treffen, um den Bergbau zu beleben, was sie in erschöpfender Weise erledigte. Unter anderem veranlaßte sie die Aufnahme neuer Grubenbetriebe (Serlo 1936). Daraufhin wurde im folgenden Jahr das Schlesische Oberbergamt in Reichenstein eingerichtet und eine revidierte Bergordnung eingeführt.

Am 18. 9. 1768 verfügte Friedrich II. die Aufnahme Gerhards als Mitglied in die Akademie der Wissenschaften (ABBA-5), was in den beiden nächsten Sitzungen der Akademie vollzogen wurde (ABBA-4). Nachdem Gerhard einige Male nicht in den wöchentlichen Sitzungen der Akademie anwesend war, zeigte er am 17. 11. 1768 Mineralstufen vor, die er von seiner Reise nach Schlesien mitgebracht hatte.
Nach diesem Zeitpunkt setzt die von P. Krusch im Jahre 1904 publizierte Darstellung der Geschichte der Bergakademie zu Berlin ein, die auf einem detaillierten Studium der preußischen Staatsakten basiert. Dieser gewissenhaften Darstellung, die die verwendeten Akten allerdings leider nicht im einzelnen benennt, wird im wesentlichen, Kürzungen und Ergänzungen einbegriffen, gefolgt.

Am 12.1.1770 erließ Friedrich II. durch den Minister und Chef der Justiz Frh. v. Fürst eine Anordnung, wonach auf allen preußischen Universitäten Mineralogie und Bergrechte ,gehörig dociret werden" sollen. Weiterhin erteilte er über den Etats-, Krieges- und dirigierenden Minister im General-Direktorium Ludwig Philipp Frh. von Hagen, der 1768 zugleich auch Chef des Bergwerks- und Hütten-Departements geworden war, dem Bergrat Gerhard den Auftrag, „den Plan zu einer vollständigen Berg-Information zu entwerfen und einen Kostenanschlag der einmaligen und laufenden Ausgaben eines besonderen Berginstitutes aufzustellen," Gerhards Plan wird von Krusch ausführlich wiedergegeben. Danach soll in fünf Halbjahren die Theorie des Berg- und Hüttenwesens, die sich auf Mathematik, Physik, Mechanik, Hydraulik, Chemie, Metallurgie, Bergbaukunde, Aufbereitungswesen, Hüttenkunde und die Lehre von den Gebirgen gründet, gelehrt werden. Im Anschluß daran sollen die Eleven noch ein Jahr auf Bergwerken zur praktischen Ausbildung verbringen.

Friedrich II. ging auf den Vorschlag Gerhards ein und akzeptierte auch die erforderlichen Geldmittel. Das zu gründende Institut erhielt nun den vorläufigen Namen ,vollständige Bergschule“. Im März 1770 wurde Gerhard nach Freiberg geschickt, um die Einrichtung der dortigen, im Jahre 1765 gegründeten Bergakademie kennenzulernen. Sein Bericht vom 24. 3. 1770 an Minister v. Hagen ist so interessant, daß er hier nach dem Original, das in den Beständen des Geheimen Staatsarchivs Preußischer Kulturbesitz überliefert ist (GSTAB-4), wiedergegeben wird:

„Da Ew. Hochfreiherrliche Excellenz mir gnädigst anbefohlen haben, mich nach denen Einrichtungen der Freibergischen im Jahre 1766 errichteten Berg Academie zu erkundigen, so habe die Ehre hierdurch unterthänigst anzuzeigen, wie bey gedachter Academie 2 Professores sich befinden, nehmlich der Berg-Rath Gellert und der Professor Charpentier. Ersterer liest die Metallurgie und Anweisung zum Hüttenwesen. Er ist zu dem Ende mit dem nöthigen Laboratorio versehen, und sind zu denen Experimenten 200 Rthl. ausgesetzet.

Der Professor Charpentier giebet Anweisung zu der practischen Geometrie, der Mechanic und der Zeichnungskunst. 
Es ist deshalb eine Anzahl von Modellen, Zeichnungen und Rissen angeschaft worden, zu deren Unterhaltung jährlich 150 Rthl. bestimmt sind.

Die ordinairen Auditores sind 6 junge Leute die auf Landesherrliche Kosten studiren und Berg Cadetten heißen. Wenn sie zu Freiberg den Cursum, so 2 Jahre dauret, absolviret, so gehen sie auf Reisen, wozu sie ein gewisses aus der Berg Zehend Casse erhalten. Es werden dazu bloß Landes Kinder und unter denen jederzeit 2 Freiberger angenommen. Wenn außer diesen Berg Cadetten noch andere hören wollen, so müssen sie bezahlen, doch werden nicht gern Fremde dazu admittiret.

Im Grubenbau wird kein Unterricht ertheilet, sondern derselbe ex Usu und bey der täglichen Befahrung derer Gruben erlernet.

Ew. Hochfreiherrliche Excellenz werden hieraus gnädigst $\mathrm{zu}$ vermerken geruhen, daß diese Einrichtung noch sehr incomplett sey, da in dem Wasser Bau, dem Forst Wesen und dem Bergbau selbst kein Unterricht gegeben, sondern der letztere bloß practisch erlernt wird, welches ein absolutes Hinderniß abgibt, daß dieses wichtige Metier je in eine ordentliche und systematische Verfaßung komme."

Gerhard geht dann in seinem Schreiben noch ausführlich auf die Köhlerei ein, die in Freiberg nicht behandelt wurde, aber höchst notwendig zu lehren wäre. Er habe sich, unter der zu ,verhoffenden gnädigen Approbation", vorgenommen, dies mit vorzutragen.

Zur weiteren Vorbereitung der neuen Ausbildungsstätte wurden geeignete Lehrkräfte ausgewählt. Es waren möglichst solche, die, zur Ersparung staatlicher Kosten, eigene Hilfsmittel bzw. Laboratorien besaßen, für die Physik der Professor des Collegiums medicum Walter (1749-1826), für die Chemie der Apotheker Valentin Rose (1762-1807), Professor de Chatillon (1747-1814) für die Mathematik und Gerhard für die Mineralogie und Berg- und Hüttenkunde. Übrigens war Gerhard keineswegs nur für die Bergakademie im Staatsdienst tätig, sondern hatte vorwiegend andere Aufgaben im Bergwerks- und Hütten-Departement zu erfüllen, auBerdem daneben noch im Ober-Bau-Departement, nachdem er zum Ober-Berg- und Bau-Rat ernannt worden war. Dadurch kam es auch da$\mathrm{zu}, \mathrm{da} ß$ er den Plan seiner Vorlesung, den er vor Eröffnung der Bergakademie, die für den 1. Juli vorgesehen war, vorzulegen hatte, erst am $7 . \mathrm{Au}-$ gust 1770 in Kleve, der preußischen Besitzung am Niederrhein, niedergeschrieben hat. Dieser Plan, der bei Krusch (1904) zusammengefaßt dargestellt ist, besteht im Original (GSTAB-5) aus einem höchst detaillierten, nur aus Stichworten bestehenden Inhaltsverzeichnis, das in $37 \mathrm{~Pa}$ ragraphen und diese wiederum in zahlreiche Unterpunkte eingeteilt ist. Der Umfang reicht von Geologie über Mineralogie, Lagerstätten- und Bergbaukunde bis zu Hüttenkunde und Technologie der Metallverarbeitung. Von Köhlerei ist darin nicht die Rede.
Da auch die anderen Lehrkräfte bereit standen, begann der Lehrbetrieb im Herbst 1770. Ihre Bezeichnung als Bergakademie wurde erst 1774 offiziell. Gerhard fing seine Vorlesungen am 1. 11. 1770 an und hatte großen Zuspruch. Unter seinen Hörern befanden sich auch $6 \mathrm{Be}$ amte, die sich weiterbilden wollten. Als Anschauungsmaterial mußte Gerhard auf seine eigenen Bestände zurückgreifen, wie die anderen Lehrkräfte auch. Die Sammlung des Bergwerksund Hütten-Departements, die sich durch Belegstücke im Zuge des Dienstbetriebs allmählich bildete, war dafür kaum geeignet.

So war nunmehr die Institution geschaffen, in der bei der weiteren Entwicklung das Königliche Mineralienkabinett entstand mit seinen systematischen und regionalen Mineraliensammlungen einschließlich der Bestände an Versteinerungen. Diese Entwicklung und die darauf folgende, die Übereignung an die Universität als Mineralogisches Institut und den Weg bis zum Museum für Naturkunde, gehört in die künftigen Fortsetzungen dieses Artikels.

\section{Verzeichnis der Archivalien}

Geheimes Staatsarchiv Preußischer Kulturbesitz Berlin-Dahlem

GSTAB-1 = I - Rep 36, Nr. 2710

GSTAB-2 $=$ I - Rep 96A, Tit $1 \mathrm{E}$

GSTAB-3 = I - Rep 121, Abt D, Tit II, Sect 1, Nr. 101, vol 1, Bl. 33

GSTAB-5 = wie vor, Bl. 117-121

GSTAB- $6=I-\operatorname{Rep} 9, \mathrm{C} 1$ b3, Fasc. 28, B1. 109

GSTAB-7 = I - Rep 96 B, Nr. 135, 6.9.1768

Archiv der Berlin-Brandenburgischen Akademie der Wissenschaften zu Berlin

ABBA-1 = I - XV, Nr. 19

ABBA-2 $=I-X V$, Nr. 32

ABBA-3 $=I-X V$, Nr. 10

ABBA-4 =I $-\mathrm{IV}, \mathrm{Nr} .32$ (Régistres de 1 'Académie)

ABBA-5 $=\mathrm{I}-\mathrm{III}, 3, \mathrm{Bl} .29$

Handschriften-Abteilung der Staatsbibliothek Preußischer Kulturbesitz Berlin

HASTB- $1=$ Mss. Boruss. fol. 740

HASTB-2 $=$ Mss. Boruss. fol. 233

\section{Schriftenverzeichnis}

Agricola, G. 1530. Bermannus sive de re metallica. Froben, Basel.

1546. De natura fossilium. Froben, Basel.

- 1556. De re metallica. Froben, Basel.

Barchatova, N. N. \& Raskin, N. M. 1976. Die Petersburger Periode der wissenschaftlichen Tätigkeit J. G. Lehmanns. - Zeitschrift für geologische Wissenschaften 4: 529-534. 
Bartnick, D. 1992. Johannes Mathesius zu St. Joachimsthal Vater der Mineraliensammler. - Mineralien-Welt 4: $55-57$.

Bernoulli, J. 1782. [Verkaufsangebot der Sammlung von A. S. Marggraf] - Leipziger Magazin zur Naturkunde, Mathematik und Ökonomie 1: 149.

Bielefeldt, E. 1980. Carl Abraham Gerhard - ein Berliner Geologe der Aufklärung. - Zeitschrift für Geologische Wissenschaften 8: $207-215$.

Boerlin, P. H. 1970. Leonhard Thurneysser als Auftraggeber. Kunst im Dienste der Selbstdarstellung zwischen Humanismus und Barock. 239 pp., Birkhäuser, Basel, Stuttgart.

Daber, R. 1970. Zur Frühgeschichte der wissenschaftlichen Sammlungen im Museum für Naturkunde an der Humboldt-Universität zu Berlin 1770-1810. - Neue Museumskunde 13: 245-255.

Degering, H. 1922. Eine Berufung an die Königliche Bibliothek im Jahre 1765/66 nach Briefen und Akten der Preussischen Staatsbibliothek. Aus der Handschriftenabteilung der preußischen Staatsbibliothek. Berlin.

Dietrich, W. O. 1960. Geschichte der Sammlungen des Geologisch-Paläontologischen Instituts und Museums der Humboldt-Universität zu Berlin. Ein Beitrag zur Paläontologie-Geschichte. - Berichte der Geologischen Gesellschaft der DDR 5: 247-289.

Döhn, H. 1988. Handschriften zur Geschichte Berlins und der Mark Brandenburg. Eine Auswahl aus den „Manuscripta Borussica" der Deutschen Staatsbibliothek. Deutsche Staatsbibliothek, Handschrifteninventare, Berlin 11.

[Eller, J. T.] 1761. Verzeichniß einer auserlesenen Sammlung vortreflicher Mineralien und Conchylien ... Berlin.

Eller, J. T. 1764. Physikalisch-Chymisch-Medicinische Abhandlungen aus den Gedenkschriften der königl. Akademie der Wissenschaften herausgezogen und übersetzt von D. Carl Abraham Gerhard, Ruediger, Berlin, Stettin und Leipzig.

Fischer, W. 1939. Mineralogie in Sachsen von Agricola bis Werner. Die ältere Geschichte des Staatlichen Museums für Mineralogie und Geologie zu Dresden (1560-1820). 347 pp. Heinrich, Dresden.

Freyberg, B. v. 1955. Johann Gottlob Lehmann (1719-1767). Ein Arzt, Chemiker, Metallurg, Bergmann, Mineraloge und grundlegender Geologe. 159 pp., Erlanger Forschungen, Reihe B, Naturwissenschaften, Erlangen.

Geyer, A. 1936. Geschichte des Schlosses zu Berlin. 1. Band. Die kurfürstliche Zeit bis zum Jahre 1698. Deutscher Kunstverlag, Berlin.

Henckel, J. F. 1725. Pyritologia oder: Kieß-Historie, als des vornehmsten Minerals, nach dessen Nahmen, Arten, Lagerstätten, Ursprung ... vom Nutzen des Bergwerks. Martini, Leipzig.

Jahn, I. 1979. Die Museologie als Lehr- und Forschungsdisziplin mit spezieller Berücksichtigung ihrer Funktion in naturhistorischen Museen. Geschichte, gegenwärtiger Stand und theoretische Grundlagen. Teil I. - Neue Museumskunde 22: $152-169$.

Krusch, P. 1904. Die Geschichte der Bergakademie zu Berlin von ihrer Gründung im Jahre 1770 bis zur Neueinrichtung im Jahre 1860. 54 pp., Verlag der Kgl. Geol. Landesanstalt und Bergakademie, Berlin.

Ledebur, L. v. 1831. Geschichte der Königlichen Kunstkammer in Berlin. - Allgemeines Archiv für Geschichtskunde des Preußischen Staates 6: 3-57.

Lehmann, J. G. 1751. Kurtze Einleitung in einige Theile der Bergwercks-Wissenschaft Anfängern zum Besten abgefasset. 192 pp., Nicolai, Berlin.

- 1756. Versuch einer Geschichte von Flötz-Gebürgen, betreffend deren Entstehung, Lage, darinnen befindliche Metallen, Mineralien und Foßilien, größtentheils aus eigenen Wahrnehmungen, chymischen und physicalischen Versuchen, und aus denen Grundsätzen der Naturlehre hergeleitet. 240 pp., Klüter, Berlin.
1758. Kurtzer Entwurff einer Mineralogie zum Dienst derer allhier in Berlin Studierenden verfaßet. 152 pp., [Berlin].

1760. Entwurf einer Mineralogie zum Dienst derer allhier in Berlin Studierenden. Zweyte und vermehrte Auflage. 150 pp., Lange, Berlin.

- 1761. Probierkunst. 318 pp., Wever, Berlin.

- 1761a. Chymische Untersuchung einer braunen Erde aus der Baumanns-Höhle auf dem Harz. - Physikalisch-chymische Schriften, als eine Fortsetzung der Probierkunst. Wever, Berlin, 358-386.

Lenz, H. O. 1856. Zoologie der alten Griechen und Römer. Becker, Gotha.

- 1861. Mineralogie der alten Griechen und Römer. Thienemann, Gotha.

Lüschen, H. 1968. Die Namen der Steine. Das Mineralreich im Spiegel der Sprache. 383 pp., Ott, Thun, München

Merkel, O. 1904. Eine Sammlung oligozäner Petrefakten aus der La-Tène-Zeit. - Jahresschrift für die Vorgeschichte der sächsisch-thüringischen Länder 3:90-93.

Moehsen, J. C. W. 1783. Leben Leonhard Thurneyssers zum Thurn, Churfürstlich Brandenburgischen Leibarztes. Ein Beitrag zur Geschichte der Alchymie, wie auch der Wissenschaften und Künste in der Mark Brandenburg gegen Ende des sechszehnten Jahrhunderts. In Beiträge zur Geschichte der Wissenschaften in der Mark Brandenburg von den ältesten Zeiten an bis zu Ende des sechszehnten Jahrhunderts. Decker, Berlin und Leipzig: $1-198$.

Morys, P. 1982. Medizin und Pharmazie in der Kosmologie Leonhard Thurneissers zum Thurn (1531-1596). - Abhandlungen zur Geschichte der Medizin und der Naturwissenschaften, Husum 43.

Neickelius, C. F. 1727. Museographia oder Anleitung zum rechten Begriff und nützlicher Anlegung der Museorum oder Raritäten-Kammern. Hubert, Leipzig und Breßlau.

[Neumann; K.] 1767. Verzeichnis einer sehr seltenen Sammlung von Kunst- und Natur-Sachen, welche 1767 in Berlin verauctionirt werden sollen. Berlin.

Nicolai, F. 1786. Beschreibung der Königlichen Residenzstädte Berlin und Potsdam, aller daselbst befindlicher Merkwürdigkeiten und der umliegenden Gegend. 3. Auflage. Nicolai, Berlin.

Niedermayr, G. 1988. „Nichts ist auf der Welt Schöneres ...". Zur Geschichte des Mineralsammelns. - Katalog der Mineralientage München 25: 2-40.

Pott, J. H. 1746-1754. Chymische Untersuchungen, welche fürnehmlich von der Lithogeognosia oder Erkenntnis und Bearbeitung der gemeinen einfacheren Steine und Erden, ingleichen von Feuer und Licht, handeln. Voss, Potsdam und Berlin.

Prescher, H. 1955. Von Sammlern und Sammlungen des Mineralreiches im XVI. Jahrhundert. In Wilsdorf, H.: Georgius Agricola 1494-1555. Zu seinem 400. Todestag 21. November 1955. Dt. Akad. Wiss., Berlin: 320-338.

Prescher, H. (Hrsg.) 1955-1992. Agricola-Gedenkausgabe (in deutschen Übersetzungen). Bde. 1-10 und Ergänzungsband. Dt. Verlag der Wissenschaften, Berlin.

Raskin, N. M. 1974. [Zur Vorgeschichte der Errichtung der Bergschule]. In Geologii Leningradskogo Gornowo Instituta. Moskwa: 9-22. [in russischer Sprache]

Reichl, O. 1930. Zur Geschichte der ehemaligen Berliner Kunstkammer. - Jahrbuch der Preußischen Kunstsammlungen 51: $223-249$.

Sander, H. 1784. Beschreibung seiner Reisen durch Frankreich, die Niederlande, Holland, Deutschland und Italien; in Beziehung auf Menschenkenntnis, Industrie, Litteratur und Naturkunde insonderheit. 683 pp. Jacobaeer, Leipzig.

Schlosser, J. v. 1908. Kunst- und Wunderkammern der Spätrenaissance. Klinckhardt \& Biermann, Leipzig.

Schmieder, C. 1807. Theophrasts Abhandlung von den Steinarten. Freyberg. 
Serlo, W. 1936. Bergmannsfamilien in Rheinland und Westfalen. Aschendorff, Münster.

Spitzer, G. 1996. ... und die Spree führt Gold. Leonhard Thurneysser zum Thurn. Astrologe - Alchimist - Arzt und Drucker im Berlin des 16. Jahrhunderts. - Beiträge aus der Staatsbibliothek zu Berlin Preußischer Kulturbesitz 3: 146 pp.

Tembrock, M. L. 1982. Eine Sammlung obereozäner, oligozäner und miozäner Mollusken aus der La-Tène-Zeit. Zeitschrift für geologische Wissenschaften 10: 1387-1391.

Thurneysser Zum Thurn, L. 1572. Pison. Das erst Theil. Von kalten/warmen minerischen und metallischen Wassern ... Eichorn, Franckfurt an der Oder.

Wilson, W. E. 1994. The history of mineral collecting. - Mineral. Record 25 (6): $240 \mathrm{pp}$.

Winter, U. 1981. Handschriften, seltene Drucke und Kuriositäten in der Churfürstlichen Bibliothek zu Cölln an der Spree. - Marginalien, Zeitschrift für Buchkunst und Bibliophilie, Berlin und Weimar 84: 50-68.

Woltersdorf, J. L. 1748. Systema minerale .. Mineral-System worin alle zum Mineral-Reich gehörige Körper ... vorgetragen werden. Berlin. 